\title{
TREATMENT OF PHIMOSIS WITH TOPICAL STEROIDS AND FORESKIN ANATOMY
}

\author{
TATIANA C. MARQUES, FRANCISCO J.B. SAMPAIO, LUCIANO A. FAVORITO \\ Urogenital Research Unit, State University of Rio de Janeiro, Rio de Janeiro, RJ, Brazil
}

\begin{abstract}
Objectives: To correlate topical steroidal treatment of stenosed foreskin with the different degrees of glans exposure and the length of time the ointment is applied.

Materials and Methods: We studied 95 patients with phimosis, divided according to the degree of foreskin retraction. Group A presented no foreskin retraction, group B presented exposure of only the urethral meatus, group $C$ presented exposure of half of the glans, and group D presented exposure of the glans, which was incomplete because of preputial adherences to the coronal sulcus. Patients were submitted to application of $0.05 \%$ betamethasone ointment on the distal aspect of the prepuce twice daily for a minimum of 30 days and a maximum of 4 months.

Results: Of 95 patients, $10(10.52 \%)$ abandoned the treatment and 15 patients in groups $\mathrm{C}$ and $\mathrm{D}$ were excluded from the study. Among the remaining 70 patients, only 4 patients $(5.7 \%)$ in group A did not obtain adequate glans exposure after treatment. In group A (38 patients), fully retractable foreskins were obtained in 19 patients (50\%) after 1 month of treatment. In group B (28 patients), fully retractable foreskins were obtained in 18 patients (64.2\%) after 1 month.

Conclusions: Treatment was successful in $94.2 \%$ of patients, irrespective of the type of foreskin anatomy. The improvement may require several months of treatment. Patients with impossibility of urethral meatus exposure present around $10 \%$ treatment failure.
\end{abstract}

Key words: penis; phimosis; anatomy; steroids; circumcision

Int Braz J Urol. 2005; 31: 370-4

\section{INTRODUCTION}

Circumcision is frequently performed in the United States and Canada, although in a variety of locations around the world, such as Europe and South America, this procedure is not done on a routine basis. When it is not done routinely, the incidence of pathological phimosis is increased (1). Pathological phimosis results when there are adherences to the fibrotic foreskin ring that make it impossible to expose the penis glans (1). This situation hinders adequate penis hygiene, which favors the occurrence of foreskin infections, repeated urinary tract infections, sexu- ally transmitted diseases and, in adults, carcinoma of the penis (2).

The correction of phimosis in infancy is performed with general anesthesia, a procedure that is not without risks, with a complication rate that may reach $34 \%$ (3). The main complications following circumcision are hemorrhage, stenosis of the urethral meatus and the foreskin ring, and even amputation of the glans (4). In addition, this procedure presents considerable costs (5).

Recently, clinical treatment of phimosis using topical corticosteroids has been proposed as an alternative to surgery with good results (6-8). Regard- 
less of the patient's age, the results are encouraging, with success rates ranging from 67 to $95 \%$ of cases $(2,8,9)$.

There are several classifications for the position of the phimotic ring $(1,2,9,10)$, although only Kayaba et al. (11) demonstrated the form and degree of retractability of the prepuce. Studies that correlate foreskin anatomy with topical treatment using corticosteroids in patients with phimosis are rare, or even inexistent.

The objective of this work is to correlate topical treatment of $0.05 \%$ betamethasone in the stenosed foreskin with the different degrees of exposure of the glans and the length of application needed for the foreskin to become fully retractable.

\section{MATERIALS AND METHODS}

Between January 2001 and October 2003, we evaluated 95 patients with phimosis for possible circumcision. The patients ranged in age from 19 months to 14 years (mean age 7.7 years). The Human Research Committee at our institution approved the investigation. An informed consent form was obtained from the parents (mother or father) of each patient.
The patients were divided into groups according to the degree of foreskin retraction (11) (Figure1). Group A consisted of patients who presented no foreskin retraction, group B presented exposure of the urethral meatus only, group $\mathrm{C}$ presented exposure of half of the glans, and group D presented incomplete exposure of the glans due to preputial adherences to the coronal sulcus.

After classification into one of the groups, the patients were submitted to application of $0.05 \%$ betamethasone ointment on the phimotic ring (distal aspect of the prepuce). Parents were instructed to gently apply traction to the foreskin until the ring appeared, applying a thin layer of cream twice daily for a minimum of 30 days and a maximum of 4 months, in association with correct hygiene of the penis. These children were followed every month in our outpatient service.

Therapy was considered successful when the prepuce was fully retractable with total glans exposure. Failure was considered when it was impossible to achieve glans exposure, when there was no alteration in the degree of stenosis after more than 4 months, and if there was infection during the treatment. In such cases, circumcision would be indicated.
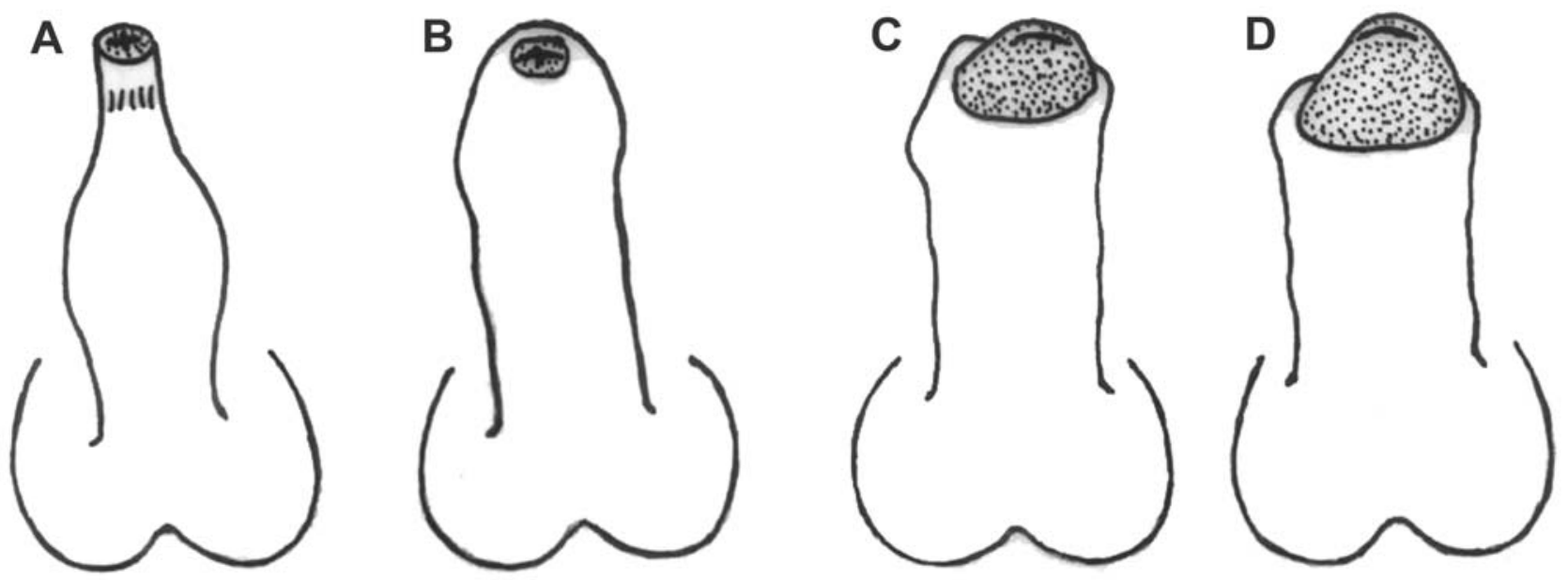

Figure 1 - Diagram based on the work by Kayaba et al. (11) showing the 4 types of foreskin according to the position of the phimotic ring and the retraction ability, as well as the incidence found in the present study. Group A - no foreskin retraction, group B - exposure of the urethral meatus, group $C$ - exposure of half of the glans, and group D - incomplete exposure of the glans due to preputial adherences to the coronal sulcus. 
For statistical analysis, we used the chisquared test. $\mathrm{P}<0.05$ indicates statistically significant differences (12).

\section{RESULTS}

The type of foreskin anatomy found in the 95 children is shown in Table-1. There was a predominance of group A (43 children - 45.2\%) and group B (34 - 35.7\%). Groups C (6 - 6.3\%) and D (12 $12.6 \%)$ presented a lower incidence. Of the 95 patients, $10(10.52 \%)$ abandoned the treatment and 15 patients in groups $\mathrm{C}$ and $\mathrm{D}$ were excluded from the study because they were not strictly considered as having phimosis. Among the patients who abandoned treatment, one presented the foreskin anatomy of group A, 6 of group B and 3 of group D. Among the remaining 70 patients, only 4 patients $(5.7 \%)$ in group A did not obtain adequate exposure of the glans after treatment.

Of the 66 patients $(94.2 \%)$ who did obtain adequate exposure of the glans after treatment (fully retractable prepuce), 38 (57.5\%) were in group A and $28(42.5 \%)$ were in group B. The response to topical treatment for the groups studied in relation to the length of time the ointment was used is shown in Table-2.

Table 1 - Incidence of the different types of foreskin anatomy found in the present study.

\begin{tabular}{lcc}
\hline Foreskin Anatomy & Patients & \% \\
\hline Group A & 43 & 45.2 \\
Group B & 34 & 35.7 \\
Group C & 6 & 6.3 \\
Group D & 12 & 12.6 \\
Total & 95 & 100 \\
\hline
\end{tabular}

In group $\mathrm{A}, 8$ patients $(21 \%)$ were $\leq 3$ years old and 30 patients $(79 \%)$ were $>3$ year old. Of the patients who responded to treatment in group A (38 of 42 patients $-90.4 \%$ ), fully retractable foreskins were obtained in 19 patients (50\%) after 1 month of treatment, in 5 patients (13.1\%) after 2 months, in 9 patients $(21.6 \%)$ after 3 months, and in 5 patients (13.5\%) after 4 months.

In group B (28 patients), 4 patients (14.2\%) were $\leq 3$ years old and 24 patients $(86 \%)$ were $>3$ year old. All patients in group B responded to treatment and fully retractable foreskins were obtained in 18 patients $(64.2 \%)$ after 1 month, in 6 patients (21.4\%) after 2 months, in 1 patient (3.5\%) after 3 months, and in 3 patients (10.7\%) after 4 months.

Independently of the group they were classified, 37 of the patients (56\%) achieved glans exposure within 30 days of treatment. Only 8 patients $(12.1 \%)$ required 4 months of treatment to obtain a fully retractable prepuce. No adverse side effects were observed from the topical betamethasone treatment. There was no statistically significant difference in satisfactory response to treatment over the course of the months between groups $\mathrm{A}$ and $\mathrm{B}$.

\section{COMMENTS}

Physiological phimosis affects $96 \%$ of newborns and its incidence diminishes with age. At 3 years old, $10 \%$ of boys present phimosis and by the age of 14 years, this incidence decreases to $1 \%$ (13).

In Australia at the beginning of the 1990s, Kikiros et al. (10) attested to the efficacy of topical corticosteroids in the treatment of preputial stenosis. Since then, several authors have shown satisfactory results (67\% to $95 \%$ ) with the topical use of betamethasone, clobetasol, sodium diclofenac, $0.05 \%$ mometasone furoate and triamcinolone acetonide (8-10).

Table 2 - Therapeutic success rate for groups A and B in relation to the length of time the ointment was applied.

\begin{tabular}{llrrrl}
\hline Groups & Month 1 & Month 2 & Month 3 & Month 4 & \multicolumn{1}{c}{ Total } \\
\hline A & $19(50 \%)$ & $5(13.1 \%)$ & $9(23.6 \%)$ & $5(13.1 \%)$ & $38(57.6 \%)$ \\
B & $18(64.2 \%)$ & $6(21.4 \%)$ & $1(3.5 \%)$ & $3(10.7 \%)$ & $28(42.4 \%)$ \\
Total & $37(56 \%)$ & $11(16.6 \%)$ & $10(15.1 \%)$ & $8(12.1 \%)$ & $66(100 \%)$ \\
\hline
\end{tabular}


Betamethasone is one of the steroids that present the best improvement rates $(13,14)$, and this was the reason the drug was used in this study. Corticosteroids act by reducing the arachidonic and hydroxyeicosatetraenoic acids in proliferative inflammatory disease of the skin, thereby inhibiting prostaglandin release and increasing the activity of dismutase superoxide. Additionally, they have the potential to release antioxidants (13). Collateral effects may occur, such as the suppression of the hypothalamus-hypophysis-adrenal axis or cutaneous atrophy. However, the doses utilized in topical treatment of phimosis are not large enough to lead to these types of complications (1). In our study, we did not observe any adverse effects in our patients.

We obtained a success rate of $94.2 \%$ from the treatment with $0.05 \%$ betamethasone ointment, which is similar to what has been found in recent studies in the literature $(1,2,13,15-18)$. All patients were advised to continue retracting the foreskin to maintain penile hygiene. We observed parent satisfaction when the decision to pursue conservative treatment was made. Topical treatment using corticosteroids has been shown to have low risk with an absence of side effects and good adherence to treatment when those responsible for the child have been well briefed.

Monthly follow-up for observation of the evolution of the phimotic ring has been shown to be fundamental in the assessment of the time at which the therapy utilized is having its effect, or whether it is ineffective. Therapy can be stopped at any time and surgery can then be indicated.

All 4 patients $(5.7 \%)$ who showed no improvement after using the ointment and required a surgical procedure were in group A. Among the patients in group A who responded to topical treatment, $35 \%$ obtained the desired result only after 3 or 4 months of treatment. The patients without any foreskin retraction (group A) presented an approximately $10 \%$ chance of not benefiting from clinical treatment, even after a long period of ointment use, and such patients will require circumcision. In group B, $70 \%$ of the patients showed the desired result within the first two months of ointment application. These results are very significant at the time of indicating the treatment, especially for patients unable to have foreskin retraction (group A), which was the most frequent situation among our patients (incidence of 45\%). Patients with foreskin anatomy in groups B presented a high chance of obtaining the desired result with treatment duration of less than 60 days.

In conclusion, topical treatment of phimosis using $0.05 \%$ betamethasone ointment presented a success rate of $94.2 \%$, regardless of the form and degree of foreskin retraction. Most previous reports have described one month of treatment; nevertheless, we found that the desired improvement might take several months of treatment.

\section{REFERENCES}

1. Orsola A, Caffaratti J, Garat JM: Conservative treatment of phimosis in children using a topical steroid. Urology. 2000; 56: 307-10.

2. Elmore JM, Baker LA, Snodgrass WT: Topical steroid therapy as an alternative to circumcision for phimosis in boys younger than 3 years. J Urol. 2002; 168: 1746-7; discussion 1747.

3. Chu CC, Chen KC, Diau GY: Topical steroid treatment of phimosis in boys. J Urol. 1999; 162: 861-3.

4. Ozkan S, Gurpinar T: A serious circumcision complication: penile shaft amputation and a new reattachment technique with a successful outcome. J Urol. 1997; 158: 1946-7.

5. Berdeu D, Sauze L, Ha-Vinh P, Blum-Boisgard C: Cost-effectiveness analysis of treatments for phimosis: a comparison of surgical and medicinal approaches and their economic effect. BJU Int. 2001; 87: 239-44.

6. Gulobovic Z, Milanovic D, Vukadinovic V, Rakie I, Perovic S: The conservative treatment of phimosis in boys. Br J Urol. 1996; 78: 786-8.

7. Wright JE: The treatment of childhood phimosis with topical steroid. Aust N Z J Surg. 1994; 64: 327-8. Erratum in: Aust N Z J Surg. 1995; 65: 698.

8. Jorgensen ET, Svensson A: The treatment of phimosis in boys, with a potent topical steroid (clobetasol propionate $0.05 \%$ ) cream. Acta Derm Venereol. 1993; 73 : 55-6.

9. Atilla MK, Dundaroz R, Odabas O, Ozturk H, Akin R, Gokcay E: A non-surgical approach to the treatment of phimosis: local non-steroidal anti-inflammatory ointment application. J Urol. 1997; 158: 196-7.

10. Kikiros CS, Beasley SW, Woodward AA. The response of phimosis to local steroid application. Pediatr Surg. Int. 1993; 8: 329-32. 
11. Kayaba H, Tamura H, Kitajima S, Fujiwara Y, Kato T, Kato T: Analysis of shape and retractability of the prepuce in 603 Japanese boys. J Urol. 1996; 156: 1813-5.

12. Sokol RR, Rohlf FJ: Biometry, 3rd (ed.), New York, USA: Freeman WH, 1995.

13. Shankar KR, Rickwood AM: The incidence of phimosis in boys. BJU Int. 1999; 84: 101-2.

14. Marzaro M, Carmignola G, Zoppellaro F, Schiavon G, Ferro M, Fusaro F, et al.: Phimosis: when does it require surgical intervention? Minerva Pediatr. 1997; 49: 245-8.

15. Lund L, Wai KH, Mul LM, Yeung CK: Effect of topical steroid on non-retractile pre-pubertal foreskin by a prospective, randomized, double-blind study. Scand J Urol Nephrol. 2000; 34: 267-9.

16. Lee KS, Koizumi T, Nakatsuji H, Kojima K, Yamamoto A, Kavanishi Y, et al.: Treatment of phimosis with betamethasone ointment in children. Nippon Hinyokika Gakkai Zasshi. 2001; 92: 619-23.

17. Monsour MA, Rabinovitch HH, Dean GE: Medical management of phimosis in children: our experience with topical steroids. J Urol. 1995; 162: 1162-4.

18. Ashfield JE, Nickel KR, Siemens DR, MacNeily AE, Nickel JC: Treatment of phimosis with topical steroids in 194 children. J Urol. 2003; 169: 1106-8.

Received: April 17, 2005

Accepted after revision: June 20, 2005

\author{
Correspondence address: \\ Dr. Luciano Alves Favorito \\ Urogenital Research Unit - UERJ \\ Av. 28 de Setembro, No. 87, fundos \\ Rio de Janeiro, RJ, 20551-030, Brazil \\ Fax: + $55212587-6121$ \\ E-mail: favorito@uerj.br
}

\section{EDITORIAL COMMENT}

These authors have confirmed successful treatment of phimosis in children with betamethasone ointment as has been shown in other studies. In addition, they have demonstrated success with lower dose betamethasone ointment $(0.05 \%$ instead of $0.1 \%)$ and that only one month treatment is needed in about half of the cases. However, they do not report on long term follow-up to determine if recurrence is a problem. Ashfield et al. (Reference 18 in article) also did not report long term follow-up but they examined patients six weeks following cessation of treatment, which should have at least detected early recurrences.

The more important point on this topic to consider is when this treatment is indicated. From the results in this study, this would seem the best treatment for phimosis causing ballooning of the prepuce with voiding and/or when phimosis is thought to be causing recurrent infections. These authors do not note that any of these boys had symptoms. While these authors and others have shown resolution of phimosis with steroid ointment, they have not demonstrated that treating asymptomatic phimosis in pre-pubertal boys has any medical benefit.

Dr. Jean G Hollowell Children's Hospital of the King's Daughters and Eastern Virginia Medical School Norfolk, Virginia, USA 